\title{
CARACTERIZAÇÃO DA MAÇÃ BRASILEIRA COMERCIALIZADA EM CURITIBA E REGIÃO METROPOLITANA
}

\author{
Retail market of Brazilian apples in \\ Curitiba and metropolitan area
}

\author{
Ricardo Cetnarski Filhoํ, Ruy Inacio Neiva de Carvalho², Vinícius Caetano Martin ${ }^{3}$ \\ ${ }^{1}$ Engenheiro Agrônomo, M. Sc., Autônomo. São José dos Pinhais, PR - Brasil. e-mail: ricardotche@hotmail.com \\ ${ }^{2}$ Engenheiro Agrônomo, Dr., Bolsista de Produtividade em Pesquisa do CNPq, Professor Titular da PUCPR, São José dos \\ Pinhais, PR - Brasil.e-mail: ruy.carvalho@pucpr.br \\ ${ }^{3}$ Engenheiro Agrônomo, Autônomo. Curitiba, PR - Brasil. e-mail: vicamar@bol.com.br
}

\section{Resumo}

A maçã (Malus domestica Bork.) é uma das frutas de clima temperado de cultivo mais extensivo no Sul do Brasil. O sucesso de um ciclo produtivo conclui-se com a boa comercialização da fruta nos principais centros de consumo. O objetivo deste trabalho foi caracterizar o mercado varejista de maçãs em Curitiba e Região Metropolitana, no período de fevereiro de 1999 a janeiro de 2000. Quatro mercados representativos na comercialização de frutas foram selecionados e visitados mensalmente para coleta de dados sobre as cultivares ofertadas, preços praticados, embalagens utilizadas, firmeza da fruta e grau de lesões na casca das frutas. Os preços reais foram corrigidos pelo INPC-IBGE para sua comparação entre os meses do ano. As cultivares de maçã comercializadas foram "Gala", "Fuji", "Golden", "Red Delicious" e outras sem a possibilidade de distinção de cultivares. A freqüência de oferta das cultivares ao longo do ano de foi de 81,3\% para a "Fuji", 72,9\% para "Gala" e "Golden" e $18,8 \%$ para a "Red Delicious". Os preços médios anuais praticados foram de R $\$ 1,58 \mathrm{~kg}^{-1}$ ("Fuji"), $\mathrm{R} \$ 1,59 \mathrm{~kg}^{-1}$ (“Golden”), $\mathrm{R} \$ 1,77 \mathrm{~kg}^{-1}$ (“Gala”) e R $\$ 2,26 \mathrm{~kg}^{-1}$ (“Red Delicious”). Todas as cultivares encontravam-se com a polpa firme e em bom estado de conservação, com apenas algumas lesões leves na casca da fruta. Concluiu-se que a maçã apresentou um período de mercado de preços mais elevados principalmente de outubro a janeiro.

Palavras-chave: Malus domestica; Comércio; Qualidade. 


\begin{abstract}
The apple (Malus domestica Bork.) is one of the temperate climate fruits more extensively cultivated in Southern Brazil. The success of a productive cycle is concluded with the good commercialization of the fruit in the main consumption centers. The objective of this work was to characterize the retail market of apples in Curitiba and its Metropolitan Region in the period from February, 1999 to January, 2000. Four representative markets in the commercialization of fruits were selected and visited monthly for data collection about marketed cultivates, sell prices, used packing-case, fruit firmness and degree of lesions on the fruits peel. The real prices were corrected by INPC-IBGE brazilian rates for their comparison along the year. The commercialized apple cultivates were "Gala", "Fuji", "Golden", "Red Delicious" and other not identified cultivates. The offer frequency of the cultivates along the year were "Fuji" with $81.3 \%$, "Gala" and "Golden" with 72.9\%, and "Red Delicious" with only 18.8\%. The annual sell prices average were $R \$ 1.58 \mathrm{Kg}^{-1}$ ("Fuji"), $R \$ 1.59 \mathrm{Kg}^{-1}$ ("Golden"), $R \$ 1.77 \mathrm{Kg}^{-1}$ ("Gala") and R\$ $2.26 \mathrm{Kg}^{-1}$ ("Red Delicious"). All cultivates had a firm pulp and good appearance, with just some light lesions on the fruit peel. The apple prices for all cultivates were higher mainly from October to January.
\end{abstract}

Keywords: Malus domestica; Market; Quality.

\title{
INTRODUÇÃO
}

A macieira é originária do oeste da Ásia e foi introduzida no Brasil com os primeiros colonizadores. Em 1969, por meio de incentivos fiscais, surgiram os primeiros pomares comerciais (ABPM, 2001). Desde esta época, a maçã sempre foi muito apreciada pelos consumidores, pois, além de possuir um sabor agradável e apetitoso, contém elementos altamente nutritivos e benéficos ao funcionamento do organismo e à saúde humana (FREY, 1990). No Brasil, as melhores condições climáticas para o cultivo da macieira encontram-se principalmente nos estados do sul, onde várias técnicas já foram desenvolvidas com o objetivo de aumentar a produtividade e melhorar a qualidade dos frutos (EBERT et al., 1998).

O cultivo da maçã no Brasil na safra 99/00 ocorreu em 32.233 ha distribuídos nos estados de Santa Catarina (52\%), Rio Grande do Sul (42\%), Paraná (5\%) e outros (1\%). A produção nacional nessa safra foi de 967.063 toneladas, com destaque aos estados de Santa Catarina (51\%) e Rio Grande do Sul (44\%). O estado do Paraná representou apenas 3\% da produção (ABPM, 2001). Salienta-se que a fruta ofertada no comércio local de Curitiba e Região Metropolitana foi, na maior parte, proveniente de outros estados.

O número de cultivares de macieira existente no mundo atualmente é muito grande, em razão dos trabalhos de melhoramento genético, utilizando-se hibridações, seleções clonais e mutações. As principais cultivares disponíveis para os produtores brasileiros nas décadas passadas foram: "Gala", "Fuji", "Golden Delicious", "Mutsu", "Starkrimson", "Royal Red Delicious", "Blackjon”, "Willie Sharp", "Melrose”, "Granny Smith", "Mollie”s Delicious", "Jonagold", "Anna”, "Rainha”, "Brasil" e outras (RIBEIRO, 1986). Outras novas cultivares já foram lançadas no Brasil como a "Eva" (IAPAR, [20-?]a), "Anabela" (IAPAR, 1997), "Carícia" (IAPAR, [20_?]b), "Baronesa" (DENARDI; CAMILO; PETRI, 1997), "Condessa" (DENARDI; CAMILO, 1998a), "Daiane" (DENARDI; CAMILO, 1998b), "Fuji Suprema" (PETRI; DENARDI; SUZUKI, 1997), "Imperatriz" (DENARDI, CAMILO, 1996) e "Lisgala"(DENARDI; CAMILO; PETRI, 1997). Mesmo com muitas cultivares disponíveis para os produtores, $91 \%$ dos pomares brasileiros foram formados com as cultivares "Gala" (46\%) e "Fuji" (45\%) (SCHMIDT; SILVA, 2000).

A cultivar "Gala" é originária da Nova Zelândia, obtida por meio do cruzamento de "Kidd"s Orange Red" com "Golden Delicious". Os frutos são de tamanho médio e uniformes, epiderme de coloração vermelha; sua conservação é regular, com tendência ao murchamento dos frutos nas condições de ambiente natural. O período de maturação dos frutos vai de janeiro a março. A cultivar "Fuji" é originária do Japão, obtida por meio do cruzamento da "Ralls Janet" com a "Delicious". Seus frutos são de tamanho médio e grande, epiderme de coloração vermelha, ligeiramente rajada e sua 
maturação estende-se até a primeira quinzena de abril. As maçãs do grupo "Golden” são originárias dos Estados Unidos da América e os frutos são de tamanho médio e grande, epiderme de coloração amareloesverdeada. A maturação dos frutos ocorre do mês de fevereiro a março (RIBEIRO, 1986).

A comercialização das cultivares de maçã é feita principalmente nas Centrais de Abastecimento e nos grandes mercados, porém poucos estudos caracterizam a comercialização local em grandes centros de consumo. Na CEASA-PR, a comercialização de maçã durante os meses de março, abril, maio, agosto, setembro e outubro é forte, com grande quantidade do produto e com tendência de preços mais baixos. Durante os demais meses, a comercialização é classificada como regular, com oferta equilibrada e tendência de preços estáveis (CEASA, [2000]).

O objetivo deste trabalho é caracterizar o mercado varejista de maçã nacional em Curitiba e Região Metropolitana no período de fevereiro de 1999 a janeiro de 2000.

\section{MATERIAL E MÉTODOS}

Foram realizadas visitas mensais em quatro mercados varejistas representativos de Curitiba e Região Metropolitana no período de fevereiro de 1999 a janeiro de 2000 para coleta de dados sobre cultivares ofertadas, preços praticados, embalagens utilizadas (forma de exposição das frutas), firmeza do fruto e grau de lesões na casca das frutas. Foram avaliadas apenas as maçãs de origem nacional. Os preços reais foram corrigidos pelo INPC-IBGE, para comparação entre os meses do ano, com base no mês de fevereiro de 1999.

As observações relacionadas à firmeza e grau de lesões na casca das frutas foram realizadas no próprio local de venda, simulando o momento em que o consumidor visualiza as frutas para a compra. Dessa forma, essas variáveis foram avaliadas enquadrando-se as frutas em um menor número de classes, visando à aproximação dos resultados obtidos com a ótica do consumidor.

Com relação à firmeza do fruto, foram utilizados os critérios: mole (imprópria para compra), pouco firme (com possibilidade de compra) e firme (ideal para compra).

As lesões foram classificadas como: graves (fruta com muitas manchas escuras na casca, imprópria para compra); médias (fruta com algumas manchas na casca, com possibilidade de compra); leves (fruta com poucas manchas na casca, boa para compra) e ausentes (fruta sem manchas na casca, ideal para a compra).

O delineamento experimental utilizado foi o de blocos ao acaso, com quatro repetições, sendo cada mercado uma repetição, e doze tratamentos, que foram as análises mensais efetuadas.

A interpretação das informações coletadas foi feita por meio de análise gráfica e a variabilidade entre os mercados em função dos meses foi avaliada pelo desvio-padrão dos dados, em vista da ausência da fruta em muitos meses e mercados.

\section{RESULTADOS}

As cultivares de maçã comercializadas no período estudado foram a "Golden", a "Red Delicious", a "Fuji" e a "Gala". Nenhuma cultivar esteve presente em todos os mercados em todos os meses do ano. A cultivar "Fuji" foi ofertada em 81,3\% das visitas, enquanto as cultivares "Golden" e "Gala" foram encontradas em $72,9 \%$ das visitas. As cultivares "Gala" e "Fuji" foram encontradas em todos os meses em apenas um mercado. A "Red Delicious" foi encontrada apenas em 18,8\% das visitas, sendo que em dois mercados não foi encontrada em nenhuma visita (TABELA 1). As cultivares "Gala" e "Fuji" representam 46\% e 45\% da produção de maçãs no Brasil, respectivamente. A cultivar "Golden" representa apenas $6 \%$ e o restante está representado por outras cultivares menos importantes (ABPM, 2001). Apesar de a maçã "Golden" ser pouco produzida em relação à "Gala", as duas foram ofertadas com igual freqüência nos mercados de Curitiba e Região Metropolitana. Mesmo em pequena quantidade, a oferta de diferentes cultivares nas estantes é uma estratégia do mercado para atrair o consumidor, oferecendo a ele maior possibilidade de escolha.

A maior parte das maçãs ofertadas nos mercados possivelmente tem origem nos estados do Sul do Brasil, pois a produção paranaense de maçãs representou apenas 3\% da produção nacional no período 
e o estado possui capacidade de armazenamento em câmaras com atmosfera controlada de apenas 11,2\% do total produzido (ABPM, 2001). Curitiba e Região Metropolitana representam um grande centro consumidor de localização mais próxima dos dois principais estados produtores de maçã, Rio Grande do Sul e Santa Catarina. A grande produção e capacidade de armazenagem de frutas nesses estados, aliada à facilidade de acesso à Região de Curitiba, garantem a oferta das maçãs em vários meses do ano.

A baixa oferta de maçãs da cultivar "Red Delicious" nos mercados da região é um reflexo da pequena importância dessa cultivar em pomares no Brasil, podendo ser encontrada, muitas vezes, em sessões especiais de frutas importadas, juntamente com pêras, ameixas e outras frutas de caroço de cultivo mais comum nos países da América do Sul, como a Argentina e Chile.

Os preços médios praticados para as maçãs "Gala", "Fuji" e "Golden" apresentaram tendência de elevação no período de setembro a janeiro, com oscilações neste período que podem estar relacionadas com o dia da visita e ofertas promocionais, típicas de grandes mercados. O período de fevereiro a maio caracterizou uma tendência de queda de preços das três cultivares, cujos preços permaneceram baixos até o mês de julho, quando passaram a apresentar nova elevação. A cultivar "Red Delicious" não foi encontrada nos meses de fevereiro, março, agosto e outubro. O preço médio mais baixo foi encontrado no mês de abril $\left(\mathrm{R} \$ 1,36 \mathrm{~kg}^{-1}\right) \mathrm{e}$ o mais alto no mês de janeiro $\left(R \$ 3,23 \mathrm{~kg}^{-1}\right)$. O preço médio anual foi de $\mathrm{R} \$ 2,26 \mathrm{~kg}^{-1}$, sendo mais elevados que os das outras cultivares, porém com as mesmas tendências de variação de preços (FIGURA 1).

A safra da maçã, normalmente ocorrida de fevereiro a abril, contribuiu para aumentar a oferta e, conseqüentemente, reduzir os preços nesse período. A boa capacidade de armazenamento das maçãs e a existência de armazenagem apropriada em muitas regiões produtoras, como Santa Catarina e Rio Grande do Sul, possibilitam a boa oferta de maçãs nos meses subseqüentes à safra, sem provocar aumento de preços. Com o passar dos meses, além da menor vida de prateleira das maçãs nos mercados, o custo agregado à fruta é maior, favorecendo a sua oferta com preços mais elevados. A precocidade de colheita (janeiro a março) e menor capacidade de conservação em câmaras frias (6 a 7 meses) da maçã "Gala" em relação à "Fuji" (RIBEIRO, 1986) pode ser o principal motivo do aumento de preço da maçã "Gala" nos meses de outubro, novembro e dezembro. A grande variação de preços da cultivar "Red Delicious" é um reflexo da sua instabilidade de oferta ao longo do ano.

A diferença de preços praticados entre os mercados em cada mês foi particular para cada cultivar. Para a maçã "Gala", o preço médio praticado nos meses de março, abril e maio foi de $\mathrm{R} \$ 1,40 \mathrm{~kg}^{-1}$, caracterizando o período de preços mais baixos. As maiores variações de preços entre os mercados foram encontrados nos meses de outubro a fevereiro, período em que o preço médio praticado foi de $\mathrm{R} \$ 2,23 \mathrm{~kg}^{-1}$, ou seja, 59,3\% maior que no período de março a maio. O preço médio anual da "Gala" foi de $\mathrm{R} \$ 1,77 \mathrm{~kg}^{-1}$ (TABELA 2).

Para a cultivar "Fuji", as variações entre os mercados foram mais constantes ao longo do ano. O preço médio anual observado foi de $\mathrm{R} \$ 1,58 \mathrm{~kg}^{-1}$. O preço mais baixo foi praticado no mês de julho $\left(\mathrm{R} \$ 1,24 \mathrm{~kg}^{-1}\right)$ e o mais alto no mês de novembro $\left(\mathrm{R} \$ 1,82 \mathrm{~kg}^{-1}\right)$, porém, mesmo nos meses de preços baixos (abril a agosto) houve maiores diferenças entre os mercados (TABELA 2).

Para a cultivar "Golden", as variações de preços entre os mercados também ocorreram ao longo do ano. No mês de novembro, ocorreu o preço médio mais baixo $\left(\mathrm{R} \$ 1,27 \mathrm{~kg}^{-1}\right)$ e em janeiro o preço médio foi mais alto $\left(\mathrm{R} \$ 2,16 \mathrm{~kg}^{-1}\right)$. O preço médio anual para esta cultivar foi de $\mathrm{R} \$ 1,59 \mathrm{~kg}^{-1}$ (TABELA 2).

Todas as cultivares foram classificadas como "firmes" ou "ideais para compra" em todos os meses e em todos os mercados. Sob o ponto de vista do consumidor, as maçãs encontravam-se sem problemas para compra em relação à firmeza da fruta, não havendo variação desta característica ao longo do ano. Mesmo nos meses em que as maçãs ofertadas são provenientes de câmaras frias, as frutas encontravam-se com boa qualidade para a venda.

A comercialização da maçã foi realizada principalmente a granel, poucas vezes foram encontradas em pacotes plásticos. Apesar de a exposição a granel das maçãs favorecer o aparecimento de danos, fato que deprecia a qualidade das frutas, principalmente as climatéricas, as lesões observadas na casca encontraram-se na faixa de leves e médias. Estes níveis de lesões não desqualificam a fruta para compra sob a ótica do consumidor, porém mesmo as lesões leves podem contribuir para a depreciação da qualidade da fruta durante o transporte e exposição nos mercados (CARVALHO, 1998). Não foram encontradas relações entre o nível de lesões, tipo de embalagem e firmeza da fruta nos meses estudados.

Rev. Acad., Ciênc. Agrár. Ambient., Curitiba, v. 6, n. 1, p. 21-27, jan./mar. 2008 
TABELA 1 - Oferta de cultivares de maçã em vários mercados varejistas de Curitiba e Região Metropolitana de fevereiro de 1999 a janeiro de 2000

Table 1 - Offer of apple cultivates at several retail market in Curitiba and its Metropolitan Region from February, 1999 to January, 2000

\begin{tabular}{|c|c|c|c|c|c|c|c|c|c|c|c|c|c|}
\hline Cultivares & Mercado & $\begin{array}{l}\text { Fev. } \\
99\end{array}$ & $\begin{array}{l}\text { Mar. } \\
99\end{array}$ & $\begin{array}{l}\text { Abr. } \\
99\end{array}$ & $\begin{array}{l}\text { Maio } \\
99\end{array}$ & $\begin{array}{l}\text { Jun. } \\
99\end{array}$ & $\begin{array}{l}\text { Jul. } \\
99\end{array}$ & $\begin{array}{l}\text { Meses } \\
\text { Ago. } \\
99\end{array}$ & $\begin{array}{l}\text { Set. } \\
99\end{array}$ & $\begin{array}{l}\text { Out. } \\
99\end{array}$ & $\begin{array}{l}\text { Nov. } \\
99\end{array}$ & $\begin{array}{l}\text { Dez. } \\
99\end{array}$ & $\begin{array}{l}\text { Jan. } \\
00\end{array}$ \\
\hline Gala & \multirow{4}{*}{ I } & $\mathrm{X}$ & $\mathrm{X}$ & & & & & $\mathrm{X}$ & $\mathrm{X}$ & $\mathrm{X}$ & $\mathrm{X}$ & $\mathrm{X}$ & $\mathrm{X}$ \\
\hline Fuji & & $\mathrm{X}$ & $\mathrm{X}$ & $\mathrm{X}$ & $\mathrm{X}$ & $\mathrm{X}$ & $\mathrm{X}$ & $\mathrm{X}$ & & & $\mathrm{X}$ & $\mathrm{X}$ & \\
\hline Golden & & $\mathrm{X}$ & $\mathrm{X}$ & $\mathrm{X}$ & $\mathrm{X}$ & & & & & & & & $\mathrm{X}$ \\
\hline Red delicious & & & & $\mathrm{X}$ & $\mathrm{X}$ & $\mathrm{X}$ & & & & & & & \\
\hline Gala & \multirow{4}{*}{ II } & $\mathrm{X}$ & $\mathrm{X}$ & $\mathrm{X}$ & $\mathrm{X}$ & & & & & & & & \\
\hline Fuji & & $\mathrm{X}$ & $\mathrm{X}$ & $\mathrm{X}$ & $\mathrm{X}$ & $\mathrm{X}$ & $\mathrm{X}$ & $\mathrm{X}$ & $\mathrm{X}$ & $\mathrm{X}$ & $\mathrm{X}$ & $\mathrm{X}$ & $\mathrm{X}$ \\
\hline Golden & & & $\mathrm{X}$ & & $\mathrm{X}$ & & $\mathrm{X}$ & $\mathrm{X}$ & $\mathrm{X}$ & $\mathrm{X}$ & $\mathrm{X}$ & $\mathrm{X}$ & $\mathrm{X}$ \\
\hline Red delicious & & & & & & & & & & & & & \\
\hline \multirow{4}{*}{$\begin{array}{l}\text { Gala } \\
\text { Fuji } \\
\text { Golden } \\
\text { Red delicious }\end{array}$} & \multirow{4}{*}{ III } & $\mathrm{X}$ & $\mathrm{X}$ & $\mathrm{X}$ & $\mathrm{X}$ & $\mathrm{X}$ & $\mathrm{X}$ & $\mathrm{X}$ & $\mathrm{X}$ & $\mathrm{X}$ & $\mathrm{X}$ & $\mathrm{X}$ & $\mathrm{X}$ \\
\hline & & $\mathrm{X}$ & & & $\mathrm{X}$ & $\mathrm{X}$ & $\mathrm{X}$ & $\mathrm{X}$ & $\mathrm{X}$ & $\mathrm{X}$ & $\mathrm{X}$ & $\mathrm{X}$ & $\mathrm{X}$ \\
\hline & & $\mathrm{X}$ & $\mathrm{X}$ & $\mathrm{X}$ & $\mathrm{X}$ & $\mathrm{X}$ & $\mathrm{X}$ & $\mathrm{X}$ & $\mathrm{X}$ & $\mathrm{X}$ & $\mathrm{X}$ & $\mathrm{X}$ & $\mathrm{X}$ \\
\hline & & & & & & & & & & & & & \\
\hline Gala & \multirow{4}{*}{ IV } & $\mathrm{X}$ & $\mathrm{X}$ & $\mathrm{X}$ & $\mathrm{X}$ & $\mathrm{X}$ & $\mathrm{X}$ & $\mathrm{X}$ & & $\mathrm{X}$ & $\mathrm{X}$ & $\mathrm{X}$ & $\mathrm{X}$ \\
\hline Fuji & & \multirow{3}{*}{$\mathrm{X}$} & \multirow{3}{*}{$\mathrm{X}$} & \multirow{3}{*}{$\mathrm{X}$} & $\mathrm{X}$ & $\mathrm{X}$ & $\mathrm{X}$ & $\mathrm{X}$ & $\mathrm{X}$ & $\mathrm{X}$ & & $\mathrm{X}$ & $\mathrm{X}$ \\
\hline Golden & & & & & $\mathrm{X}$ & $\mathrm{X}$ & & $\mathrm{X}$ & & $\mathrm{X}$ & & $\mathrm{X}$ & \\
\hline Red delicious & & & & & & $\mathrm{X}$ & $\mathrm{X}$ & & $\mathrm{X}$ & & $\mathrm{X}$ & $\mathrm{X}$ & $\mathrm{X}$ \\
\hline Presença mensal & $(\%)$ & 62 & 62 & 56 & 75 & 62 & 56 & 62 & 50 & 56 & 56 & 68 & 62 \\
\hline
\end{tabular}

TABELA 2 - Variação mensal do preço médio de maçãs das cultivares "Fuji”, "Golden” e "Gala" comercializadas no mercado varejista de Curitiba e Região Metropolitana, preços médios corrigidos pelo INPC-IBGE com base em fevereiro de 1999

Table 2 - Month variation of average price of apple cultivates "Fuji", "Golden" and "Gala" in the retail market in Curitiba and its Metropolitan Region, average prices corrected by INPC-IBGE based on February, 1999

\begin{tabular}{|c|c|c|c|c|c|c|}
\hline \multirow[t]{2}{*}{ Mês/ano } & \multicolumn{2}{|c|}{ "Fuji" } & \multicolumn{2}{|c|}{ "Golden" } & \multicolumn{2}{|c|}{ "Gala" } \\
\hline & $\mathbf{R} \$ \mathrm{~kg}^{-1}$ & s* & $\mathbf{R} \$ \mathrm{~kg}^{-1}$ & s & $\mathrm{R} \$ \mathrm{~kg}^{-1}$ & $\mathbf{s}$ \\
\hline Fev/99 & 1,67 & 0,13 & 1,93 & 0,41 & 1,37 & 0,32 \\
\hline Mar/99 & 1,79 & 0,04 & 1,47 & 0,14 & 1,08 & 0,06 \\
\hline $\mathrm{Abr} / 99$ & 1,54 & 0,39 & 1,43 & 0,15 & 1,39 & 0,38 \\
\hline Mai/99 & 1,32 & 0,20 & 1,29 & 0,21 & 1,22 & 0,07 \\
\hline Jun/99 & 1,30 & 0,26 & 1,44 & 0,24 & 1,41 & 0,07 \\
\hline Jul/99 & 1,24 & 0,34 & 1,34 & 0,11 & 1,49 & 0,06 \\
\hline Ago/99 & 1,38 & 0,31 & 1,61 & 0,17 & 1,63 & 0,10 \\
\hline Set/99 & 1,64 & 0,10 & 1,49 & 0,22 & 1,87 & 0,05 \\
\hline Out/99 & 1,73 & 0,22 & 1,73 & 0,24 & 2,49 & 1,06 \\
\hline Nov/99 & 1,82 & 0,05 & 1,27 & 0,71 & 2,34 & 0,67 \\
\hline Dez/99 & 1,78 & 0,34 & 1,92 & 0,18 & 3,05 & 0,48 \\
\hline Jan/00 & 1,82 & 0,50 & 2,16 & 0,28 & 1,88 & 0,40 \\
\hline Média & 1,58 & & 1,59 & & 1,77 & \\
\hline
\end{tabular}

$*_{\mathrm{s}}=$ desvio-padrão 


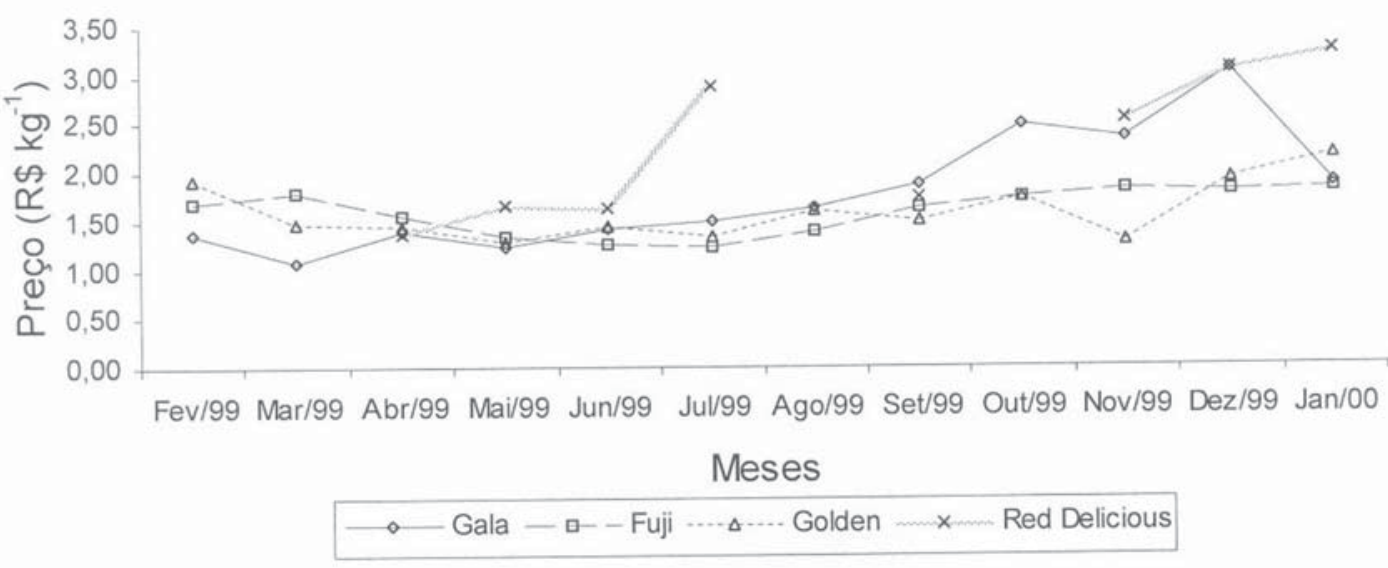

FIGURA 1 - Preço médio das maçãs "Gala", "Fuji", "Golden" e "Red Delicious" comercializadas no mercado varejista de Curitiba e Região Metropolitana de fevereiro de 1999 a janeiro de 2000

Figure 1 - Average price of apple cultivates "Gala", "Fuji", "Golden" and "Red Delicious" in the retail market in Curitiba and its Metropolitan Region from February, 1999 to January, 2000

\section{CONCLUSÕES}

Os preços médios anuais praticados para a maçã "Red Delicious" foram os mais altos

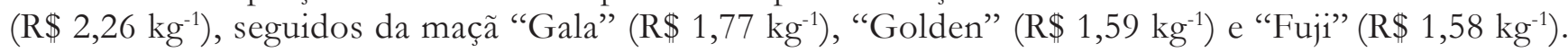

A cultivar de maçã mais encontrada nos mercados foi a "Fuji" (81,3\%), seguida da "Gala" (72,9\%), "Golden" (72,9\%) e "Red Delicious" (18,8\%).

A principal forma de comercialização da maçã nacional foi realizada a granel. venda da fruta.

As lesões na casca encontraram-se na faixa de lesões leves e médias, ainda possibilitando a

Todas as cultivares tiveram sua firmeza classificada como "firme" em todas as visitas, em todos os mercados.

\section{REFERÊNCIAS}

ABPM. Informações estatísticas. Disponível em: <http://www.macabrasileira.com.br/informacoes.htm>. Acesso em: 29 maio 2001.

CARVAlHO, R. I. N. Colheita de frutas. In: CARVALHO, R. I. N. (Coord.). Produção de frutas em pomar doméstico. Curitiba: Champagnat, 1998.

CEASA-PR. Calendário de comercialização de produtos hortigranjeiros na CEASA-Curitiba. Curitiba: DITEC, [2000]. 3 p.

DENARDI, F.; CAMILO, A. P. Epagri 404-Imperatriz-Nova cultivar de macieira. In: CONGRESSO BRASILEIRO DE FRUTICULTURA, 4., 1996, Curitiba. Anais... Curitiba: Sociedade Brasileira de Fruticultura, v. 14. p. 470. 1996.

. Epagri 406-Baronesa: nova cultivar de macieira. de maturação tardia para o Sul do Brasil.

Revista Brasileira de Fruticultura, Cruz das Almas, v. 19, n. 2, p.185-189, 1997.

Epagri 408-Condessa: nova cultivar de macieira. de baixa exigência em frio hibernal. Agropecuária Catarinense, Florianópolis, v. 11, n. 2, p. 12-15. 1998a. 
Daiane: nova cultivar de macieira para colheita em março. Agropecuária Catarinense, Florianópolis, v. 11, n. 3, p. 6-8. 1998 b.

DENARDI, F.; CAMILO, A. P.; PETRI, J. L. Epagri 407-Ligala: mutação da cultivar de macieira Gala com epiderme mais colorida. Agropecuária Catarinense, Florianópolis, v. 10, n. 1, p. 5556, 1997.

EBERT, A. et al. Raleio de frutos em macieira no Alto Vale do Rio do Peixe em Santa Catarina. Florianópolis: EMPASC, 1998. p. 65.

FREY, W. Fraiburgo: berço da maçã brasileira. Curitiba: Vicentino, 1990.

IAPAR. Macieira IAPAR 77 carícia. Curitiba: Instituto Agronômico do Paraná, [20—?]a. 6 p. (Folheto de divulgação).

IAPAR. Macieira IAPAR 75 EVA. Curitiba: Instituto Agronômico do Paraná, [20—?]b. 6 p. (Folheto de divulgação).

IAPAR. Macieira IAPAR 76 Anabela. Curitiba: Instituto Agronômico do Paraná, 1997. 6 p. (Folheto de divulgação).

PETRI, J. L.; DENARDI, F.; SUZUKI, A. Epagri 405-Fuji Suprema: nova cultivar de macieira. Agropecuária Catarinense, Florianópolis, v. 10, n. 3, p. 48-50. 1997.

RIBEIRO, P. A. Descrição e comportamento de algumas cultivares de macieira no Sul do Brasil. In: Manual da cultura da macieira. Florianópolis. EMPASC. 1985. cap. 4, p. 59-91.

SCHMIDT, S.; SILVA, A. L. Maçã: renova-se a exigência pela qualidade. Informativo SBF, Jaboticabal, Ano 18, n. 3, p. 4-5, 2000.

Recebido: 13/02/2007

Received: 02/13/2007

Aprovado: $31 / 10 / 2007$

Approved: 10/31/2007 